\title{
Dynamiques des français populaire africains : état des faits, état de la recherche et prospective
}

\author{
Camille Roger Abolou \\ Université de Bouaké \\ abolou_cr@yahoo.fr
}

\section{Introduction}

En Afrique noire francophone, en marge des français standards aux singularismes topolectaux, se développe un autre type de français, non standard ou basilectal (Ploog, 2002) ${ }^{1}$, le français populaire africain (désormais FPA), résultat de contacts entre le français et les langues africaines, dans les grandes villes africaines telles Abidjan, Dakar, Libreville, Ouagadougou, Kinshasa, Yaoundé, Douala, etc. Pratiqué par des classes sociales défavorisées, dans les quartiers populaires et précaires, il s'étend, de nos jours, aux journalistes, politiques, intellectuels dans les situations de communication particulières. Son étude constitue un point de mire de nombreux chercheurs et un domaine épistémologique en friche, bouillonnant, excitant sur bien des plans géographique (vaste étendue géographique qu'il couvre), historique (depuis la colonisation), méthodologique (les différentes approches historiques, anthropologiques, linguistiques, sociolinguistiques), théorique (fécondité des concepts et méthodes), etc. Cette curiosité scientifique de ce début du 21e siècle semble magnifier un équilibre sain entre préoccupations théoriques et constitution de corpus (Abolou, 2007 ; Blanchet, 2000 ; Dalbéra, 2002 ; Meunier-Crespo 1994; Wald, 1990; etc.), considérations géopolitiques et individuations sociolinguistiques (Blanchet, 2004 ; Chaudenson, 2000 ; Lafage, 1998; etc.), économie linguistique et changement linguistique (Martinet, 1975 ; Manessy, 1995 ; Mufwene, 2005, 2001 ; Nicolaï, 2001 ; etc.), de même qu'un déséquilibre malsain entre descriptions linguistiques et prospective sociolinguistique.

Les divers travaux définissant les FPA comme les produits d'une catégorisation sociale, semblent recommander une posture typologique et non écolinguistique.

La typologisation des FPA s'est imposée comme une doxa, dans de nombreux travaux, par un foisonnement d'outils méthodologiques tels que : la catégorisation (Wald, 1994 ; etc.), la polynomisation (Marcellesi et al., 1974 ; etc.), l'individuation (Blanchet, 2004, 2000 ; etc.), la nomination-frontiérisation (Canut, 2000 ; Feussi, 2008, 2006 ; etc.), etc. Pour Marcellesi et Gardin (1974: 231) l'individuation linguistique constitue un «... ensemble des processus par lesquels un groupe social acquiert un certain nombre de particularités de discours qui peuvent permettre de reconnaître, sauf masquage ou simulation, un membre de ce groupe. ». Alors que Blanchet (2004 : 32) s'interroge sur l'émergence des variétés dans la complexité des situations sociolinguistiques ainsi :

\begin{abstract}
L'individuation est le processus par lequel émerge l'identification d'une unité (socio) linguistique (« multiplexe », donc), en tant que constituant une entité reconnue distincte d'autres ensembles (socio) linguistiques, possédant des attributs spécifiques globaux qui ne se limitent pas à la somme des attributs de ses parties. Ce processus aboutit à la reco (n) naissance de cette entité (le tiret est volontaire, puisqu'une langue ne «naît» que lorsqu'elle est « reconnue » en tant que telle). Ce processus est de type glottopolitique (c'est-à-dire qu'il relève de facteurs sociopolitiques associés aux pratiques linguistiques) et non strictement linguistique.
\end{abstract}

Les FPA sont, par conséquent, confrontés à des casse-têtes méthodologiques ${ }^{2}$ comme : investir les identités linguistiques stigmatisées dans la catégorisation sociolinguistique ; définir la responsabilité des linguistes face aux catégories établies sur les usages des locuteurs légitimes ou non légitimes, lettrés ou non lettrés, francophones «réels» ou «occasionnels»; construire des objets langues face aux représentations individuelles (causées par le procès d'urbanisation) ou collectives des locuteurs. 
Or, l'approche écolinguistique (Calvet, 1999 ; Mufwene, 2005, 2001 ; etc.) a pour but, non des moindres, de rechercher les modèles évolutionnistes des écosystèmes du FPA caractérisés singulièrement par les locuteurs, les usages, les représentations voulues ou perçues dans un contexte de plurilinguisme généralisé empreint de malaise diglossique. Les FPA peuvent être ainsi considérés, à la suite de Mufwene (2005), comme des espèces, ontologiquement différents, phylogiquement semblables. Dès lors, ils incitent à re-évaluer les approches multiples qui ne se construisent pas dans des relations d'équivalence entre catégorisants et catégorisés mais dans les niches écolinguistiques pourvoyeuses, en paraphrasant Wald (1994 : 117), d'éléments déclencheurs tels la légitimité du locuteur, la compétence communicative, l'adéquation locale et l'intentionnalité. Ces éléments permettent l'émergence des FPA au travers de leur blasonnement social et identitaire dans un contexte, sans cesse, marqué par l'intrication des facteurs démographiques, géographiques, sociologiques, technologiques, didactiques, etc.

Faire l'état des lieux (l'évaluation critique des postures théoriques, le re-questionnement des modes d'objectivation des FPA, etc.) se révèle nécessaire pour une autre écologie de l'évolution des FPA.

\section{Les français populaires africains : données et modèles}

Les FPA, complexes et dynamiques, sont des variétés du français d'Afrique qui donnant à voir un un ordre chaotique (Robillard, 2007, 2001, 2000, 1998). Ils brouillent les constructions identitaires, instaurent la diversité des dénomminations, masquent la représentativité des corpus en territoire urbain et se révélent comme des multiplexes sociolinguistiques (Blanchet, 2000). Je retiendrai trois modèles hypothétiques : les franco-véhiculaires, les argots et les franco-bâtards.

\subsection{Les franco-véhiculaires : le modèle du français populaire ivoirien}

Les villes africaines sont des lieux d'hétérogénéité, de dynamiques sociales qui ont permis l'examen de l'émergence des franco-véhiculaires - français populaires servant de véhiculaires interethniques dans les pays n'ayant de véhiculaire africain - parmi lesquels le français populaire ivoirien (désormais FPI) semble être la variété la plus représentative (Calvet, 1991; Chaudenson, 1989; Lafage, 1980, 2002 ; Manessy, 1989 ; Valdman, 1978 ; etc.). En Côte d'Ivoire, le FPI est à la croisée de plusieurs facteurs tels que : social (les catégories socio-professionnelles), sociolinguistique (les usages et les représentations linguistiques), démographique (l'immigration, l'exode rural), géographique (la macrocéphalie d'Abidjan), politique (les politiques publiques), etc. représentés graphiquement dans le schéma ci-dessous (Abolou, $2007: 121)$ :

Figure 1 : Représentation graphique du réseau social du FPI

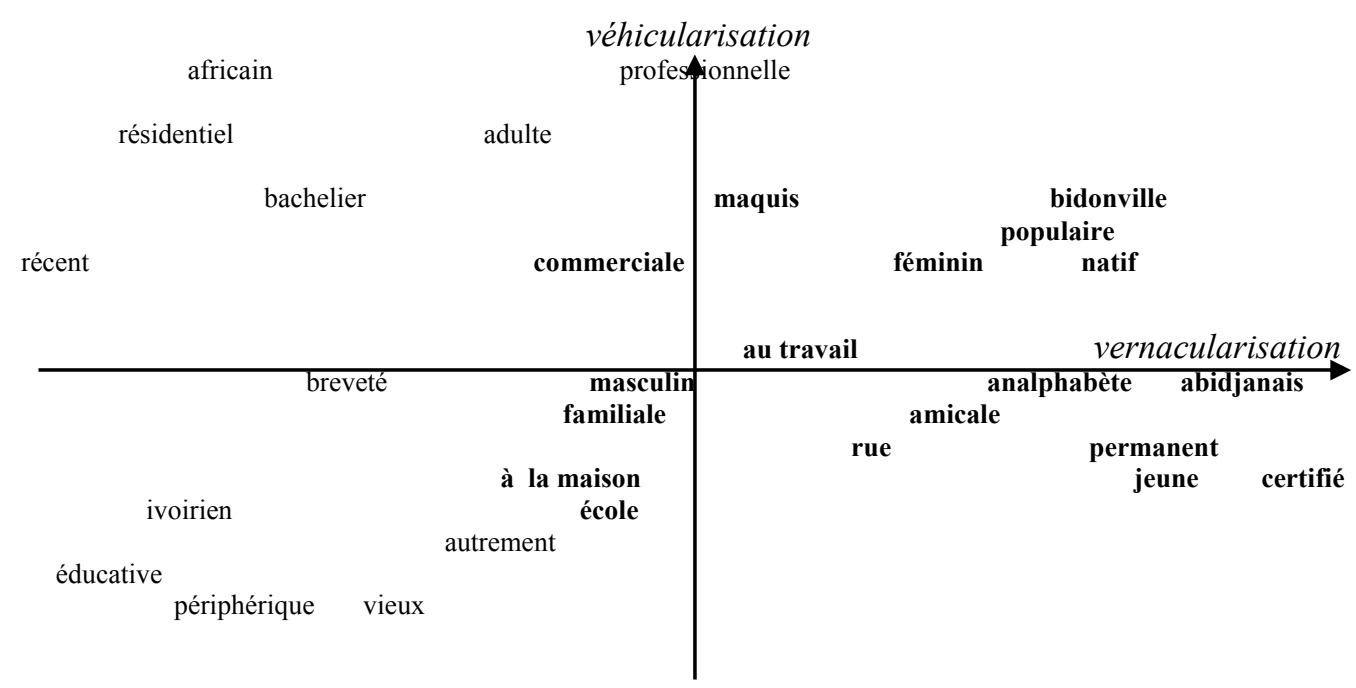


La configuration des facteurs (items lexicaux représentés dans le graphique) est la conséquence directe et/ou médiate de la manière dont les Ivoiriens en général, les Abidjanais en particulier, s'approprient le FPI. A la lecture du graphique, deux réseaux sociaux s'en dégagent: le centre du réseau (items en caractère gras) et la périphérie du réseau (items en caractère maigre). Au centre du réseau (dont les items sont en caractère gras), les locuteurs adultes, en majorité analphabètes, vivant longtemps à Abidjan, dans les quartiers populaires et bidonvilles, utilisent le FPI dans les maquis (lieux populaires de restauration) et dans les rues, etc. Lafage (1998 : 137) conclut en ces termes: "la rue devient le vrai centre de "formation/création" de français véhiculaires et le lieu principal d'apprentissage» A la périphérie, des locuteurs adultes, lettrés, vivant dans les quartiers périphériques, utilisent le FPI à la maison, à l'école.

L'urbanisation des villes africaines a créé des réseaux informels, lieux de revendications, de rejets, d'adhésions communautaires, garantis par les acteurs sociaux en proie à un équilibre social. A ce titre, ils développent des relations égalitaires prises en charge par les discours entre pairs et commerciaux. Ces discours I reflètent la diversité linguistique des locuteurs dans la mesure où ils se nourrissent de l'apport et de l'influence des langues africaines par le truchement des figures, des images, des expressions, etc. Du fait de l'importance des flux migratoires entre le Côte d'Ivoire et la sous région, par exemple, le FPI s'est africanisé en franchissant les frontières des pays voisins grâce aux jeunes, étudiants, élèves, ouvriers agricoles, manœuvres et employés de maison, de même qu'aux musiques urbaines, au théâtre populaire, etc. (Napon, 1992). Dès lors, le FPI est une langue identitaire dont le choix conscient et délibéré s'inscrit dans une diglossie réseautée s'apparentant à une stratégie de contournement de la langue officielle, le français et des politiques publiques de rationalisation des acteurs sociaux. Témoins les expressions qu'on peut entendre au Mali, au Burkina Faso, en Guinée, etc., comme : «C'est l'homme qui a peur, sinon, y a rien », « y a foye » (foye du Jula signifiant rien), « Tchogo tchogo, ça va aller », « Tchogo nan Tchanga » (on va s'en sortir), etc. Ce qui a conduit bien des auteurs (Abolou, 2008, 1994 ; Boutin, 2002 ; Hattiger, 1983 ; Jabet, 2006 ; Knutzen, 2002 ; Lafage, 2002, 1998 ; Lescutier, 1985 ; Ploog, 2007 ; etc.) à adopter des hypothèses diverses et variées allant de la pidginisation/véhicularisation à la créolisation/vernacularisation qui se sont imposées, depuis les années 1970, aux linguistes comme révélatrices d'un boom intellectuel dans les études des langues en évolution.

\subsection{Les argots : les modèles du camfranglais et du nouchi}

Depuis quelques années, dans les réseaux urbains, des argots apparaissent comme des réponses linguistiques particulières aux inégalités sociales, urbaines et communicationnelles (Calvet, 1994; Goudailler, 2003 ; François-Geiger, 1989 ; etc.). Langue métissée (Sessep N'sial, 1979 ; etc.), mélange de langue (Wijnands, 2005 ; etc.), langue hybride (Lafage, 1998; Queffélec, 2008, 1998; etc.), langue mixte, etc. : telles sont les désignations classiques portées sur ces argots en terrain africain. Ils sont définis en général comme des langues qui se mélangent de manière hétéroclite ou qui empruntent ses composantes morphologiques, lexicales, syntaxiques à une langue tierce. Calvet (1999: 136) les appréhende de la manière suivante :

Toute une série de faits témoigne de cette dernière fonction, en particulier l'apparition, dans différents pays, d'argots dans lesquels domine parfois le lexique français et d'autres fois un lexique issu d'une ou de plusieurs langues africaines. C'est ainsi qu'en Côte d'Ivoire se développe le nouchi..., qu'on utilise un argot spécifique au Burkina Faso, au Sénégal, au Mali, au Congo Kinshasa [...], etc.

A la suite de Calvet (1999), je distinguerai, pour le besoin de la modélisation decriptive, deux types d'argot: l'argot mixopéte et l'argot mixofuge. L'argot mixopète est un argot dont les ressources linguistiques (morphologie, lexique, syntaxe et sémantaxe, etc.) proviennent en partie des langues africaines. L'argot mixofuge est un argot dont plus de la moitié des ressources linguistiques est issue des langues européennes (français, anglais, espagnol). Les qualificatifs mixofuge et mixopète, loin des débats ayant alimenté la vision mixofuge de la linguistique historique et comparée, apparaissent ici comme des concepts purement opératoires à cerner la «mixité » des argots africains. Car, dans les situations 
francophones, ces argots peuvent être repérés sur le continuum francophone de l'intercompréhension allant de la rupture à la continuité de l'intercompréhension. Un francophone comprendra mieux le camfranglais que le nouchi. De plus, à la suite de Mufwene (2002:51), le développement des langues mixtes s'établit par le choix de la langue cible parmi les langues de contact. En terrain africain, certains argots auront pour langue cible le français, d'autres les langues africaines. Toutes ces précautions méthodologiques m'amènent à distinguer deux modèles d'argot : le camfranglais et le nouchi.

\subsubsection{Le camfranglais : un argot mixofuge}

La situation sociolinguistique du Cameroun, pays multilingue avec deux langues officielles, l'anglais et le français qui se superposent à près de 250 langues locales et au pidgin-english, a favorisé, outre le français local (Biloa, 2003 ; Kiessling, 2005 : Mendo-Ze, 1997; etc.), l'émergence d'un argot mixofuge, dénommé « camfranglais » dans les principales ville camerounaises (Bamenda, Buea, Douala, Yaoundé, etc.). Le camfranglais, mot composé de l'affixe «cam» du Cameroun auquel s'ajoute «franglais» dont les particules « -fran » et « -glais » signifient respectivement « français » et « anglais », est un parler composite et protéiforme (Ntsobé, 2008) qui a bénéficié, à la lecture de nombreux travaux, de deux parcours d'objectivation : partir de la langue objet à la mise en œuvre des usages linguistiques et partir des productio langagières à la construction identitaire d'une langue objet. La premier parcours, plus ancien, met en relief le camfranglais que bien des auteurs (Biloa, 2003; Echu, 2001; Essono, 1997 ; Feral, 2006, 2004 ; Fosso, 1999 ; Ntsobé, 2008 ; etc.) considèrent comme un code autonome dérivant du français «makro » (parler des voyous, des marginaux, etc.). Le camfranglais, du fait de sa complexité, va conduire ces auteurs à interpeler sa faisabilité descriptive ou les modalités de recueil et de transcription de ses données. Le second parcours, emprunté par Harter (2007), Feussi (2008), etc., s'appuie sur un processus de construction identitaire qui insinue l'altérité linguistique, plus loin l'altérolinguistique (Robillard, 2007).

L'étude du camfranglais met ainsi en jeu deux approches: l'appropriation linguistique et le constructivisme linguistique. Les appropriationnistes (Biloa, 2003, 1999; Echu, 2001 ; Feral, 2006, 2004, 1998 ; Ntsobé, 2008 ; etc.) définissent le camfranglais comme un mélange de français, de langues autochtones camerounaises et de pidgin-english qui s'est développé par appropriation des normes scolaires du français et/ou de l'anglais. Le camfranglais a une syntaxe française ou anglaise (Biloa, 1999) et un stock lexical comprenant en majorité des lexèmes français, anglais et pidgin-english qui ont subi des changements morphologiques (troncation, métathèse) ou sémantiques. Les constructivistes, s'inscrivant rigoureusement dans l'altérité linguistique, cernent le camfranglais - appelé aussi francanglais - comme un jeu de construction identitaire (Feussi, 2008, 2006 ; Schroder, 2003 ; Harter, 2007 ; etc.) dans les villes francophones et anglophones. Ce qui a amené Feussi (2008:33) à interroger ses pratiques et ses représentations linguistiques :

\footnotetext{
La place du francanglais dans la société camerounaise est d'autant plus paradoxale que le regard porté sur ce parler, bien que péjoratif pour l'essentiel, n'empêche qu'il s'ancre de plus en plus profondément et au quotidien dans les pratiques linguistiques des Camerounais. (...) Son statut emblématique ne souffre d'aucune contestation sociale.)
}

L'altérolinguisique est au centre de nombreux travaux contemporains où les représentations précèdent les pratiques langagières. Le camfranglais semble évoluer vers une autonomisation plus sécuritaire qu'usaïque concurrençant de facto le pidgin-english dans ses loci anglophones.

\subsubsection{Le nouchi : un argot mixopète}

Le nouchi est un argot mixopète dont plus de $90 \%$ du lexique provient des langues ivoiriennes en occurrence le dioula, le baoulé, le bété, etc. Il est parlé en général par les jeunes de/dans la rue, scolarisés ou déscolarisés, dans les situations sociales « formelles » (cours de recréation des lycées et collèges, etc.) et informelles (rues, gares routières, «fumoirs» (lieux de consommation des stupéfiants), vidéoclubs, 
quartiers populaires, etc.) ? Ce qui a amené Kouadio (1992 : 142) a égrené les conditions sociales de son émergence de cette variété en ces termes :

\begin{abstract}
...le nouchi est né au début des années 80 . Et cette date est loin d'être fortuite. Il surgit au moment où les jeunes que l'école ivoirienne rejetait dans la rue par dizaine de milliers chaque années et ce depuis 1975 arrivaient à maturité et choisissaient justement la rue comme leur territoire.
\end{abstract}

Le nouchi, de nos jours, gagne de plus en plus une population locutrice importante qui se recrute dans toutes les couches sociales de la société abidjanaise. Dès lors, son statut semble être éclaté sous forme de fractales identitaires validées par les espaces urbains particuliers, lieux de représentations, de croyances communes, en somme de symbolisation d'un univers subculturel entre les communautés ethnolinguistiques et la communauté francophone.

Les premiers travaux ont été signalés vers le milieu des années 1980 avec Ahua \& Coulibaly (1986) et Gouédan (1988). C'est dans les années 1990 que Lafage (1991), Ahua (1996), Kouadio (1992) n'ont pas hésité, selon les besoins méthodologiques et les prouesses théoriques du moment, à le désigner diversement «français de rue », "argot des lycéens », etc. La fonctionnalisation des pratiques langagières (médiation linguistique, communication interethnique, vernaculaire des jeunes âgés de 10 à 30 ans [Kouadio, $2006: 177$ ], cacologies, etc.), la complexification des rapports interlinguistiques (nouchi/FPI) ont conduit au recueil des corpus dans les réseaux informels (Kouadio, 2006 ; Kubé, 2005, 2002 ; Lafage, 1998 ; etc.). La plupart des auteurs recourent aux approches plus lexicographiques que morphosyntaxiques. Selon l'approche lexicographique, le nouchi marque son originalité et sa singularité « légendaire » dans ses ressources lexicales particulières composées essentiellement d'emprunts aux langues locales. Ces ressources lexicales, stéréotypes culturels, semblent dépeindre les données socioculturelles des communautés linguistiques en présence. Selon l'approche morphosyntaxique, nombre de travaux ont révélé que la quasi totalité des lexèmes ne varient ni en genre, ni en nombre, ni en personne. De même, le nouchi ne fonctionne pas selon les principes syntaxiques propres. Il puise à trois sources syntaxiques : un peu de la syntaxe du français standard local (selon le continuum nouchiphone : nouchi acrolectale des intellectuels et politiques, nouchi mésolectal des peu scolarisés), beaucoup plus de la syntaxe du FPI et des langues locales. A telle enseigne que les classes syntaxiques semblent disparaître au profit d'un stock lexical "imagé » circulant sans grand risque d'une classe à une autre (exemples : en prédication, on aura : Ils ont train la go "Ils ont violé la jeune fille» ou «La jeune fille a été violée »; en détermination, on aura : Le train a béhou «Le train est parti »). Le nouchi se situerait, par conséquent, dans une zone de transgressions caractérisée par les omissions du forclusif, du pronom impersonnel il, de la négation ne, du pronom relatif qui, de l'emploi de l'adverbe mal à valeur intensive, du déictique là postposé, etc.

De plus, certains auteurs (Ahua, 2007 ; etc.) se sont interrogés sur la place du nouchi dans les médias scripturaux et électroniques (bandes dessinées, Internet). Les besoins de communication écrite ont instauré une diversité de codes graphiques d'un média à un autre. Pour Ahua (2007: 184), la graphisation du nouchi s'impose au travers d'un code orthographique dont les raisons sont dans son acuïté dans certains pays africains et européens (diaspora ivoirienne) et les nouvelles technologies de l'information et de la communication (TIC) :

Le nouchi, aujourd'hui, à l'heure de l'Internet, souffre du manque d'un code graphique. Une écriture pour que ses usagers puissent «tchatcher à l'aise», en exprimant toutes les potentialités que leur langue possède! Un système graphique pour que ces Ivoiriens et ceux qui en sont également fascinés arrivent à « tchêkê » sur le ckavier de leur ordinateur par exemple et envoyer un «vrai» message, contenant toutes leurs intentions, à leurs destinataires.

La popularité des argots africains s'accorde plus, selon certains auteurs (Bulot, 2004 ; etc.) qui n'ont pas hésité à leur appliquer les concepts de territorialisation et de stigmatisation, aux conditions socio-spatiales 
(les quartiers populaires, les gares routières, les milieux scolaires, les enfants de la rue, les jeunes, etc.), économiques, politiques, artistiques (les chansons populaires, les musiques urbaines, etc.) et médiatiques (les bandes dessinées, les sites web, etc.) qui sous tendent les lieux intelligibles de leur manifestation.

\subsection{Les franco-bâtards : les modèles du franlof et du fransango}

Les franco-bâtards (frangache, franco-fulfulde, franco-lingala, francolof, fransango, etc.), selon Wijnands (2005: 11), sont : "... des parlers adultérins du français qui n'ont pas toujours de noms propres... .Il est même à parier que les idiomes franc-bâtards soient plus nombreux...». Ils apparaissent comme des franco-Xphoniques qui s'enracinent dans les communautés ethnolinguistiques urbanisées de nombre de pays africains. Ils ont reçu des approches définitionnelles variées et des dénominations diverses (codeswitching, code-mixing, alternance codique, alternance des codes, alternance des langues, métissage linguistique, discours mixte, etc.). Bien des auteurs (Dreyfus, 1995; Queffélec, 1998; Thiam, 1994 ; Wénézoui-Deschamps, 1995, 1994, 1988; etc.) se sont rués vers leur description. Se distinguant nettement de l'emprunt et de l'interférence, les franco-bâtards se particularisent par l'alternance codique c'est-à-dire le «changement ou [l'] alternance de langues ou de variétés linguistiques dans un discours ou une conversation » (Baylon, 1991:152) selon la règle de la contrainte de Poplack (1988). Parmi les travaux, le franlof, appelé aussi francolof et le fransango ou franc-sango ont été abondamment instrumentalisés comme des modèles heuristiques car stables, collectifs et reconnus socialement.

\subsubsection{Le franlof et le malaise diglossique}

Le franlof a émergé à Dakar dans un contexte de malaise diglossique. En tant que mélange de français et de wolof, il est l'apanage des étudiants et des intellectuels sénégalais, dénotant ainsi un mode de reproduction sociale miné par un bilinguisme élitaireI

À Dakar, le plurilinguisme s'organise essentiellement autour de trois langues : la langue véhiculaire, le wolof, les langues des migrants (manding, diola, peul sérère) et la langue officielle, le français. Parlé par 35\% de locuteurs à Dakar (Calvet, 1994: 210), le français, langue de l'Etat, de l'éducation et des médias, bénéficie d'un statut nettement surévalué par rapport à son corpus. Le wolof, utilisé par $95 \%$ de dakarois, est la langue véhiculaire qui s'impose dans toutes les situations sociales allant du centre ville commercial et administratif à la périphérie. Le wolof, au corpus surévalué en comparaison à son statut, occupe les fonctions secondaires du français. Les langues des migrants sont des langues grégaires qui sont parlées en famille, à la périphérie de Dakar. Elles subissent dans les échanges familiaux (parents/enfants) les contraintes communicationnelles du wolof urbain. Cette situation sociolinguistique donne à découvrir des diglossies enchâssées dans lesquelles le wolof devient à la fois variété haute et basse selon les latitudes fonctionnelles des langues en présence à Dakar.

Avec l'émergence du franlof, le plurilinguisme dakarois va se reconfigurer autour de deux pôles fédérateurs, le français et le wolof, deux véhiculaires associés à l'urbanité et spécialisées respectivement à l'écrit et à l'oral.

Figure 2 : représentation de la situation sociolinguistique de Dakar

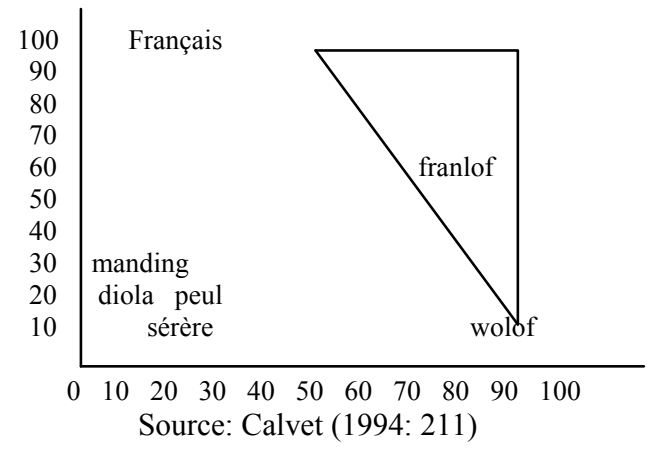


Le franlof joue un rôle de remplissage de fonctions complémentaires du français et du wolof. D'après les descriptions des auteurs comme Daff (1995), Ndao (1996), Dreyfus (1995), Swigart (1992), etc., il est une variété partagée par un nombre de plus en plus important de locuteurs jeunes et adultes, scolarisés ou non, à la maison, à l'école, au chantier, dans les médias audiovisuels (STV, STV2, etc.), etc. Thiam (1994 : 50) décrit ses mécanismes en ces termes : « ... les locuteurs recourent au mélange des deux codes [...] de manière telle que la compétence en français du sujet parlant apparaisse comme étant au moins égal à sa compétence en wolof.» Le franlof, en tant que discours mixte, peut être francisé par les scolarisés ou «wolofé » par les non scolarisés. Lorsqu'il est francisé ou «wolofé », les locuteurs recourent respectivement à certains emprunts wolof et français. Le passage du code non marqué (français) au code marqué (wolof) traduit les dispositifs symboliques de la construction d'une réalité urbaine, réitère la complexité constitutive du franlof vis à vis du non « franlophone » et encense le bilinguisme inégalitaire.

\subsubsection{Le fransango et le bilinguisme égalitaire}

La République Centrafricaine (RCA) a produit, principalement à Bangui, un franco-bâtard aux usages métissés dont les manifestations signifiantes sont empreintes de l'intelligibilité des bilingues banguissois face à une concurrence déloyale français/sango dans les situations formelles et non formelles. Cette situation a transformé le fransango en langue « adultère » et l'a imposé comme "deuxième maitresse » (une métaphore de deuxième langue acquise que j'ai forgée) des banguissois, une population urbaine estimée à $27,9 \%$ dont les francophones réels et occasionnels représentent respectivement $9,2 \%$ et $18,7 \%$ (Chaudenson, 2000).

Le marché linguistique de Bangui est ainsi marqué par la concurence de deux langues, le français et le sango. La domination du sango véhiculaire a suscité chez les jeunes scolarisés, selon Wald (1984: 81), une alternance de code omniprésente dans les contextes sociaux ordinaires. Cette alternance a interpellé Bouquiaux (1969:64) en ces termes :

En fait, on aboutit à cette situation paradoxale qu'il est impossible de tracer la frontière entre l'emploi conscient de l'une ou l'autre langue, le sango faisant très largement appel aux emprunts français et le français parlé par les locuteurs sango se plaque sur les structures rudimentaires de celui-ci. La plupart des locuteurs ne se rendent pas compte eux-mêmes du passage de l'un à l'autre.

Ainsi, les analyses linguistiques du fransango (Bouquiaux, 1969 ; Wénézoui-Deschamps, 1995, 1994 ; etc.), pour la plupart, tracent deux processus de changement structurel qui s'excluent mutuellement: la regrammaticalisation du français et la relexification du sango. La relexification du sango est un modèle de genèse dans lequel les lexèmes français subissent la régularisation des paradigmes morphologique déjà existante du sango. Dans ce modèle, le fond lexical français reste plus ou moins visible et constant dans les productions langagières. Dès lors, il demeure opaque et incompréhensible pour les non fransangophones. Dans la regrammaticalisation du français, l'adaptation des lexèmes à la morphologie sango favorise la régularisation des lexiques acclimatés voire reconstitués culturellement. Pour Wald (2004), Wenezoui (1988), etc., le fransango regrammaticalise le français à l'aide des marqueurs syntaxiques sango tels les déterminants ( $\grave{a}, s \grave{o}, n \grave{l}$, etc.), les marques aspecto-temporelles (a, awe, etc.), les pronoms personnels ( $m b i, m o$, etc.), les présentatifs (yeke "être»), etc. A telle enseigne qu'au niveau de la détermination, l'ordre progressif du français devient régressif (exemple : ce libanais devient libanais so). Au niveau de la prédication, les flexions verbales $\mathrm{du}$ français tombent au profit des marques aspecto-temporelles (exemples : la production de diamant augmente se traduit par production ti diamant ti e a augmenter; Il a arrêté la parole en question se traduit par lo arrêter tene ni awe). Wénézoui-Deschamps (1995:149) souscrit en ces termes : 
Le fransango, par conséquent, tire ses potentialités fonctionnelles du sango et ses catégories lexicales du français. Il se présente ainsi syntaxiquement comme une variété du sango et lexicalement comme une variété du français.

Ces modèles de FPA peuvent être évalués à la lumière de l'écologie de l'évolution des langues. Car, selon Martinet (1975: 6) «L'existence d'une constante évolution pose, pour toutes les sciences du comportement, la nécessité de distinguer entre la description de structures synchroniques et l'examen des conditions de l'évolution ... ». Cette nécessité de distinction exige deux points de vue : la linguistique interne et la linguistique externe. La linguistique interne met l'accent sur l'analyse synchronique préalable d'une langue avant toute formulation d'hypothèses diachroniques. La linguistique externe prend appui sur la fonction d'une langue avant toute formulation d'hypothèses synchroniques. Ces deux visées sont confrontées, à vrai dire, aux écosystèmes des FPA dans lesquels la notion d'économie, en tant que « [...] recherche permanente de l'équilibre entre les besoins contradictoires qu'il faut satisfaire: les besoins communicatifs d'une part, inertie mémorielle et inertie articulatoire d'autre part ... » (Martinet, 1975 : 178) revêt une importance capitale.

\section{Ecologie de l'évolution des français populaires africains}

Les FPA évoluent de manière divergente parce qu'ils fonctionnent dans des niches écolinguistiques différentielle. Ploog (2007), parlant du FPI, en donne les raisons de la manière suivantes :

La complexité de la tâche d'élaboration discursive est fonction du réseau de communication dans lequel elle s'accomplit : plus la constellation sociale est instable, plus les refontes des modalités de communication sont nombreuses; plus une communauté est hétérogène, plus les dynamiques langagières que l'on y observe sont marquées. En cela, il revient au non-standard un rôle central dans les changements linguistiques.

Cette clarification méthodologique semble présider au cautionnement du modèle chaudensonnien, modèle de prospective des situations francophones, dans l'appréciation des scénarios d'évolution des FPA. Selon ce modèle, les FPA apparaissent dans la francophonie occasionnelle. La francophonie occasionnelle est au corpus ce que la francophonie réelle est au statut. Le corpus en régression constante dans la plupart des pays africains constitue un vivier écolinguistique où l'on peut cerner les systèmes déclencheurs de l'évolution des FPA.

Figure 3 : Représentation graphique de la francophonie occasionnelle

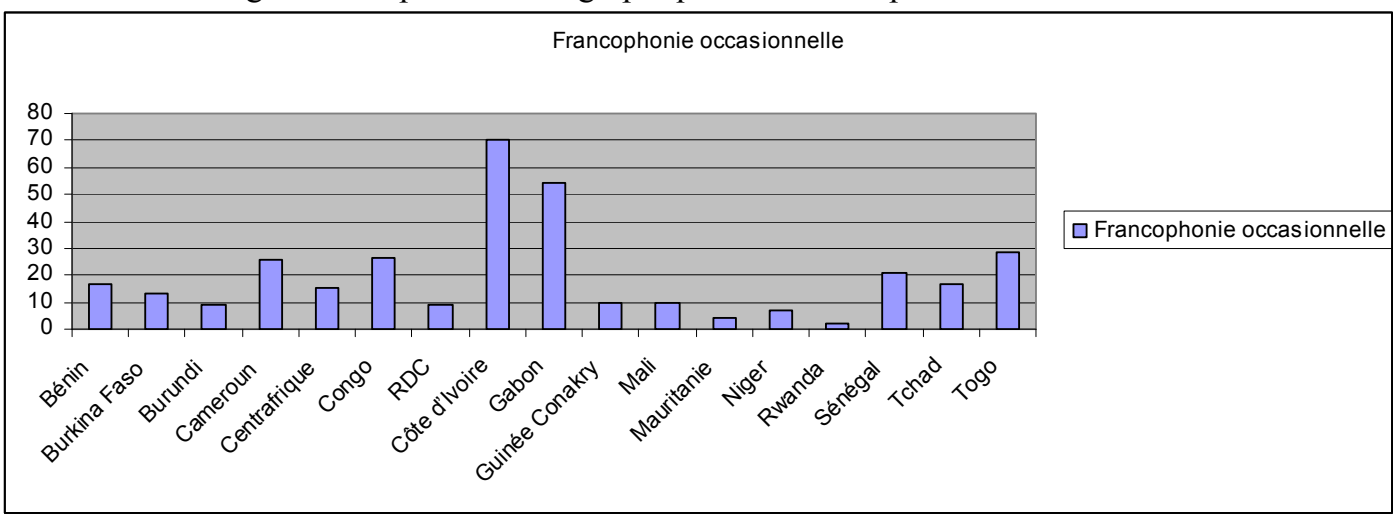

Soucrce : Chaudenson (2000)

Selon ce graphique, deux grandes niches s'en dégagent selon les pays : la niche des francovéhiculaires (Côte d'Ivoire, Gabon) et la niche des franco-bâtards (tous les autres pays). Entre 
ces deux niches, il est des niches intermédiaires qui surgissent dans la plupart des pays francophones, la niche des argots, scorie écolinguistique de la croissance urbaine.

\subsection{La niche des franco-véhiculaires}

Le multilinguisme/multiculturalisme a développé en Afrique noire francophone une niche des franco-véhiculaires où le français apparaît comme un véhiculaire interethnique. Dès lors, les franco-véhiculaires s'imposent comme des langues polysystémiques ou cosystémiques défiant la typologie linguistique classique de Schleicher et autres.

Les franco-véhiculaires semblent prospérer en Côte d'Ivoire, au Gabon, etc. En Côte d'Ivoire, par exemple, c'est le cas du FPI parlé dans la sous région, dans les pays ayant un nombre important de leurs compatriotes (Burkina Faso, Mali, Guinée, etc.). Ces franco-véhiculaires, systèmes linguistiques aux particularités phonologique, morphologique, lexicale, syntaxique, prosodique, sémantique, seraient des realia caractéristiques à la société, au locuteur (âge, sexe, groupe, catégorie socioprofessionnelle, etc.) et à l'espace urbain. Ils se déterritorialisent sous la poussée de l'immigration, du mariage mixte, de l'urbanisation et de l'industrialisation. Selon Boutet (1997: 50), ce sont des langues déterritorialisées, car œuvrant dans des situations de communication multiples et connexes telles les restaurants, les marchés, les rues, les bars, les familles élargies, etc., en somme les espaces de quotidien (Balandier, 1992 ; Gumperz, 1989 ; Goffman 1973 ; Hall, 1978 ; Maffesoli, 1979 ; etc). Les capitales africaines sont devenues des marchés d'enculturation, de transculturation, de chosification des individus où les identités sociales sont planifiées dans le sens d'un conformisme urbain, d'une rentabilisation des plans de développement urbain.

\subsection{La niche des franco-bâtards}

La niche des franco-bâtards est une niche où le français est en concurrence avec des langues africaines qui remplissent des fonctions secondaires. Les franco-bätards se manifestent comme des réponses linguistiques à des besoins identitaires. Le poids de l'image sociale des locuteurs pèse sur les façons de parler des bilingues africains dans les grandes villes africaines.

\subsection{La niche des argots}

La niche des argots prend source dans les réseaux sociaux pris en charge par les locuteurs en quête d'une identité linguistique dans un univers urbain africain, théatre des changements sociaux, culturels, économiques, etc. Les argots apparaissent comme des refuges identitaires pour les jeunes. Il en résulte des phénomènes d'hybridation linguistique lourds de conséquences sur le continuum francophone de l'intercompréhension. La croissance urbaine des pays africains [Bénin (Cotonou), Cameroun (Douala), Centrafrique (Bangui), Congo (Brazzaville), Sénégal (Dakar), etc.] a érigé ces argots africains comme symbole d'une urbanité linguistique (Bulot, $2008,2004)$ et de l'échec des systèmes éducatifs africains.

\section{Modélisations des scénarios d'évolution}

A partir des niches écolinguistiques que j'ai esquissées «grossièrement», modéliser les scénarios d'évolution des FPA est une gageure. Car, on pouvait considérer ces niches comme des systèmes chaotiques empêchant la prédictibilité de l'évolution des FPA. Cependant, à la suite de Nicolai (2001), l'on peut se donner des critères des tendances évolutives tels que: l’hétérogénéité, la théâtralité, la variabilité et l'historicité. 


\subsection{Le scénario de l'alternance codique}

Le premier scénario, plus ancien, met l'accent sur l'antériorité de l'alternance codique dans tout processus de changement linguistique en Afrique noire francophone. L'émergence d'une langue mixte comme résultat, croisement de deux langues A et B qui ont coexisté et se soient fondues en une nouvelle forme, est fondée sur la cristallisation d'un mélange de code impliquant au préalable une situation variable de plurilinguisme, un milieu social hétérogène et clivé, et des processus d'appropriation. L'analyse synchronique, à partir de l'observation méticuleuse des discours mixtes, des alternances linguistiques, permet de rendre compte des tendances évolutives des franco-bâtards vers des argots dand le schéma ci-dessous :

\section{Alternance codique $>$ Langue mixte}

\subsection{Le scénario de la mixité linguistique}

Le deuxième scénario, récusant cette succession logique, prend appui sur la fonctionnalisation des FPA dans les réseaux de communication. Une langue mixte peut apparaître dans un réseau de communication pour devenir un sociolecte générationnel, puis un véhiculaire africain (Queffélec, 2009). Dans ce scénario, l'alternance codique n'a pas droit de cité. Une langue mixte, dont l'historicité fait défaut, se révèle dans les situations de communication non formelles comme langue identitaire : c'est le cas de l'hindoubil en RDC, du camfranglais au Cameroun et du nouchi en Côte d'Ivoire. Ce sont aussi des langues en danger qui peuvent disparaître par l'action conjointe des politiques publiques de scolarisation, d'alphabétisation, d'interdiction des pratiques sociales « souteraines », etc.

\section{Langue mixte $>$ Réseaulecte $>$ sociolecte générationnel}

\subsection{Le scénario de la véhicularisation/vernacularisation}

Le troisième scénario semble établir, à chaque stade d'évolution, un équilibre entre le besoin de communication et les usages linguistiques. Deux grandes tendances sociolinguistiques se signalent : la véhicularisation et la vernacularisation. La tendance à la véhicularisation s'entrevoit dans l'usage des FPA comme des véhiculaires interethniques. La tendance à la vernacularisation est marquée par le dynamisme des espaces d'initiatives et de certains espaces résidentiels tels les quartiers populaires et bidonvilles, par l'informalisation croissante des pratiques quotidiennes (éducation informelle, économie informelle, etc.) [Lauter, 1991 ; Leimdorfer et Marie, 2002] où l'on assiste à une régularisation formelle des FPA (Calvet, 1999; Dumond, 1995, 1991). Prenant l'exemple du FPI, Ploog (2007: 2-3) reconstruit le scénario du véhicularisation/vernacularisation : installation du pouvoir colonial (1893-1933) [alternance français/langues Kwa], expansion urbaine (1933-1951) [apparition des mécanismes de code-switching (communicatif/palliatif)], le miracle ivoirien (1951-1975) [émergence du FPI, langue de communication interethnique], émergence de l'ivoirité (19851997) [vernacularisation du FPI], etc.

\section{Alternance codique $>$ Langue mixte $>$ véhiculaire $>$ vernaculaire}

\subsection{Le scénario de la pidginisation/créolisation}

Le quatrième scénario est une version révisée du troisième scénario par application des concepts de pidginisation/créolisation. Car, la véhicularisation est à la vernacularisation ce que la pidginisation est à la créolisation.

$$
\text { Alternance codique }>\text { langue mixte }>\text { pidginisation }>>>\text { créolisation ?!! }
$$

Le FPI est devenu un prétexte épistémologique pour les créolistes. Une lecture des différentes postures permet de cerner le scénario pidginisation/créolisation en terrain africain (mis à part les créoles urbains, 
historiquement formés) ${ }^{3}$. Chez Lafage $(1980: 10)$, le FPI est décrit comme un phénomène de pidginisation de la manière suivante :

... dans la mesure où non seulement cette langue est largement répandue par la scolarisation dont elle est le vecteur, mais encore où elle assure la fonction véhiculaire interethnique pour une importante fraction des masses analphabètes, sous la forme, il est vrai, d'une variété pidginisée et peut-être en voie de créolisation.

La pidginisation du FPI recommande ainsi l'aspect sociodémographique (la situation de contact, l'immigration, la scolarisation et l'analphabétisme) et l'aspect sociolinguistique (la fonction véhiculaire). Privilégiant la succession logique pidginisation/créolisation versus véhicularisation/vernacularisation, Lafage (2002) semble tracer une " petite » voie de vernacularisarion/créolisation dans certains quartiers populaires d'Abidjan où les enfants d'immigrés acquièrent le FPI comme langue première. Pour Hattiger (1983), par exemple, le FPI est un pidgin avancé qui se restructure de trois façons :

- La restructuration interne est une restructuration propre aux créoles où les systèmes linguistiques du FPI comme le syntagme nominal (absence de déterminant) s'élaborent sur le modèle des créoles conservateurs ;

- La restructuration sur le substrat est une restructuration qui peut s'observer au niveau du pluriel, du possessif, du complétif, du distributif et du relatif. Selon Hattiger (1983:290), les langues locales ivoiriennes joueraient un rôle primordial sur «[...] le modèle offert par [...] le baoulé et le dioula. »;

- La restructuration sur la norme académique est une espèce de «[...] réabsorption de la variété pidginisée par la forme standard. » (Hattiger, 1983 : 291).

Toutes ces restructurations se focalisent sur le syntagme nominal. Manessy (1989:141-142), quant à lui, partant d'une réflexion sur la norme, aborde le problème de la pidginisation du FPI en ces termes :

\begin{abstract}
Il se crée des habitudes, des façons de dire qui, dans la mesure où les usagers y attachent une valeur particulière, celle d'indice d'appartenance au groupe par exemple, tendent à se fixer en règles ; tel nous avons décrit sous le nom de "vernacularisation" à propos du français populaire d'Abidjan et qui est susceptible d'introduire quelque cohérence dans l'infinie diversité des variantes individuelles. Il y aurait là, par combinaison entre la refonte du système grammatical et la résurgence de modes de structuration sémantique proprement africains, l'amorce d'une créolisation du français, si le mouvement n'est puissamment freiné par le prestige et l'omniprésence [...] du français des lettrés.
\end{abstract}

Le FPI, appropriation de la norme exogène, se caractérise essentiellement par les tendances à la dilution des classes sociales, à la neutralisation des préjugés scolaires. Ce sont des tendances évolutives qui projettent le FPI sur une voie de créolisation possible dont les référents théoriques sont à rechercher dans la théorie d'approximation de Chaudenson (1989). En fait, pour Manessy (1994, 1989, etc.), le FPI, norme endogène puissante, est une approximation d'approximation du français standard local. 
Le FPI, selon les créolistes généralistes et descriptivistes (Abolou, 2008, 1997, 1994 ; Chaudenson, 2004, 1989 ; Hattiger, 1983 ; Valdman, 1978 ; etc.), se créoliserait. Ainsi, pour Valdman (1978), le FPI peut être considéré comme un « créole » du fait des conditions externes liées à l'emploi multilatéral, à la forte immigration et à l'absence d'une langue véhiculaire ivoirienne répandue et des conditions internes qui ont trait aux phénomènes de la simplification interne, de la réduction de la forme interne et de la stabilité relative. Se focalisant sur des corpus écrits et oraux, Valdman (1978) dégage quelques traits caractéristiques du FPI communs aux créoles classiques tels que : l'absence de distinction de genre et de pluriel, l'agglutination du déterminant du nom, la réduction morphologique, l'emploi du fonctionnel. Le FPI se distingue des pidgins français classiques par son haut degré d'élaboration syntaxique mis en relief dans la postposition du déictique là (Abolou, 2007 ; Ploog, 2007), dans l'emploi des marques se et $i$ (lui se manger «il a mangé », le démon i penser «Le diable est rusé »), des pronoms personnels de forme tonique moi, toi, lui (moi content toi « je t'aime»), etc. Tous ces traits ont conduit Valdman (1978:47) à se justifier ainsi :

Si nous nous accordons avec Duponchel pour refuser au FPA le statut de pidgin et y voir un gradient linguistique représentant divers degrés d'approximations au français [...] nous n'excluons pas la possibilité qu'il puisse se transformer en une langue autonome. Il serait alors non pas un pidgin, puisque son rôle dépasserait celui de permettre la simple intercompréhension dans des situations de rapports transitoires et s'étendrait à la plupart des fonctions cognitives, expressives et identificatrices des langues naturelles.

Pour Abolou $(2008,1994)$, le FPI est un « créole » à tradition urbanocratique. Cette hypothèse repose sur la prise en compte des réseaux de communication dans le procès de créolisation en terrain africain. Si les créoles des caraïbes se différencient de ceux de l'océan indien c'est que les problèmes de communication sont nettement différents et les réponses linguistiques spécifiques varient selon les réseaux sociaux. La réponse linguistique, pouvant engendrer des réseaux formels ou informels, installerait, grosso modo, deux modèles de créolisation : le modèle de créolisation à tradition plantocratique et le modèle de créolisation à tradition urbanocratique. Dans le modèle plantocratique, le réseau formel véhicule des informations opérationnelles qui sont des informations de nature verticale et descendante entre le maître et l'esclave. Bentolila $(1987: 118)$ souscrit en ces termes :

L'initiative du discours, dans le cas du créole haïtien, revenant automatiquement au maître, l'énoncé de l'esclave n'étant qu'un écho servilement modulé de l'énoncé à lui adressé. Les échanges étant réduits au strict minimum, touchant d'une part à l'indication des tâches, d'autre part à l'affirmation de l'effectuation de la tâche.

A telle enseigne que le système linguistique des créoles, plus précisément le système aspecto-temporel, est fortement marqué par ce type d'information. En créole haïtien, par exemple, l'opposition 0/AP, selon Bentolila (1987: 119) stigmatise les rôles sociaux et les modes d'énonciation : «La marque AP assertorique marquant le discours du maître avec une valeur injonctive, la marque zéro apodictique marquant le discours-écho de l'esclave avec une valeur de constat d'effectuation ». Ainsi, il apparaît toutà-fait plausible que, dans les sociétés à grande économie de plantation, le mode de communication descendante aurait fortement affecté le système linguistique créole. Dans le modèle urbanocratique, le réseau informel est un réseau qui produit des informations «motivantes", identitaires, se manifestant sous forme de rumeurs, d'échanges d'opinion, de faits, de représentations, etc. Il développe des relations égalitaires, des échanges entre pairs. Le mode de communication latérale qui en découle a généré un système linguistique ressemblant à celui des créoles conservateurs. Le réseau informel aurait installé une créolisation urbanocratique particulière depuis le $20^{\circ}$ siècle. Chaudenson (1989: 22), malgré ces « hypothétiques » ressemblances, nie catégoriquement toute approche visant à considérer le FPI comme un créole en ces termes :

Le français populaire d'Abidjan, par exemple, présente des traits qui le rapprochent des créoles ; on ne saurait le considérer comme tel car il ne présente pas les caractères "'historiques" et sociolinguistiques que comporte la définition que nous proposons des créoles. 
Pour lui, le FPI, ne bénéficiant pas des mêmes circonstances historiques $\left(17^{\text {ème }}\right.$ et $18^{\text {ème }}$ siècle), géographiques (îles) et sociales (société esclavagiste avec les maîtres, les esclaves et les bossales [ayant fonction d'encadrement et ne parlant que créole]), peut être considéré comme un français marginal et non un créole.

Ces quatre scénarios, résultat d'une procédure d'abstraction, semblent recommander trois modèles d'évolution des FPA dans le tableau ci-dessous :

Tableau 1 : Modèles d'évolution du FPA

\begin{tabular}{|l|c|c|c|c|}
\hline \multirow{2}{*}{$\begin{array}{l}\text { Modèles } \\
\text { d'évolution }\end{array}$} & Phase 1 & Phase 2 & Phase 3 & Phase 4 \\
\cline { 2 - 5 } & $\begin{array}{c}\text { Alternance } \\
\text { codique }\end{array}$ & Langue mixte & Véhiculaire/Pidgin & Vernaculaire/Créole \\
\hline Modèle 1 & + & + & + & $+?$ \\
\hline Modèle 2 & - & + & $+?$ & - \\
\hline Modèle 3 & + & $+?$ & - & - \\
\hline
\end{tabular}

Le modèle 1 met en place une succession logique des phases évolutives. C'est un modèle inhérent aux franco-véhiculaires comme le FPI. Le modèle 2 déploie une rupture de succession liée à la complexité des conditions d'émergence des argots, moins nombreux et restrictifs, pouvant disparaître (l'hindoubill a disparu dans les conditions peu élucidées [Edéma, 200].) ou évoluer vers les pidgins en fonction des restructurations externes et internes (politique, économique, sociale, culturelle, éducative, etc.). Le modèle 3 concerne les franco-bâtards. C'est un modèle d'évolution très aléatoire du fait des paramètres externes qui fluctuent en fonction des politiques éducatives, linguistiques et publiques.

\section{Conclusion}

Les FPA connaissent, de nos jours, une évolution fulgurante, l'informalisation des pratiques urbaines aidant. Utilisés par la plupart des Africains, dans les échanges entre pairs, en famille, dans les cours de recréation, au marché, dans les rues, dans les gares, dans les quartiers populaires, etc., ils sont en train d'enrichir pour les uns et/ou de se défaire pour les autres (d') un espace francophone par leur portée sociale et identitaire. Ces contextes d'usage ont produit des problématiques diverses imposées par les contraintes du milieu urbain et les visées scientifiques qui incitent à la modélisation des scénarios de leur évolution. De ces scénarios, les FPA semblent évoluer différentiellement selon les niches écolinguistiques de l'univers urbain africain.

\section{Références bibliographiques}

Abolou, C. R. (1994). De la sociolinguistique à la syntaxe. La créolisation du français populaire d'Abidjan: descriptions, analyses et évaluation. Thèse de doctorat unique. Paris : Université Renée Descartes - Paris V.

Abolou, C. R. (1997). Crises épistémologiques en créolistique. Généralistes, descriptivistes : un clivage à dépasser dans l'étude de la créolisation. Repères, $\mathrm{n}^{\circ} 1,13-30$.

Abolou, C. R. (2007). De l'apport de l'information statistique à la constitution des corpus des français populaires africains. Le cas du français populaire d'Abidjan. Le français en Afrique, ${ }^{\circ} 22,107-126$.

Abolou, C. R. (2008). La marque là et ses implications syntaxique et sociolinguistique en français populaires africains et en créoles à base lexicale française. Cahiers du CERLESHS, n²9, 1-24.

Ahua, B. et Coulibaly, A. (1986). Langage branché d'origine inconnue. Fraternité Matin, 3 sept, 2-3.

Ahua, B. et Coulibaly, A. (2006). La motivation dans les créations lexicales en nouchi. Le français en Afrique, $\mathrm{n}^{\circ} 21$, 143-157.

Ahua, M. B. (1996). L'argot des lycéens d'Abidjan. Mémoire de maitrise, Université de Cocody, FLASH.

Ahua, M. B. (2007). Élaborer un code graphique pour le nouchi : une initiative précoce ?. Le français en Afrique, $\mathrm{n}^{\circ}$ 22, 183-198.

Ahua, M. B. (2008). Mots, phrases et syntaxe du nouchi. Le français en Afrique, $\mathrm{n}^{\circ} 23,135-150$.

Balandier, G. (1992). Sociologie du quotidien. Encyclopedia Universalis, 1188-1191.

Baylon, Ch. (1991). Sociolinguistique, société, langue et discours, Paris : Nathan.

Bentolila A. (1987). Marques aspecto-temporelles en créole haïtien : de l'analyse synchronique à la formulation d'hypothèses diachroniques. Diogène, $\mathrm{n}^{\circ} 137,69-52$. 
Billiez, J. et Rispail, M. (dir) (2003). Contacts de langue. Modèles, typologie, interventions. Paris : L'Harmattan.

Biloa, E. (1999). Structure phrastique du camfranglais. In Echu, G. \& Grundstrom, A. W. (eds), Official Bilinguilism and linguistic Communication in Cameroon, New-York: Peter Lang, 117-174.

Biloa, E. (2003). La langue française au Cameroun. Berne : Peter Lang.

Blanchet, P. (2000). La linguistique de terrain. Méthode et théorie. Une approche ethno-sociolinguistique. Rennes : PUR.

Blanchet, P. (2004). L'identification sociolinguistique des langues et des variétés linguistiques : pour une analyse complexe du processus de categorization fonctionnelle. Paris : MIDL, 31-36.

Bouquiaux, L. (1969). La créolisation du français par le sango véhiculaire, phénomène réciproque. Annales de la Faculté des Lettres et Sciences Humaines de Nice, 7, 57-70.

Boutet, J. (1997). Langage et société. Paris : Seuil.

Boutin, B. A. (2002). Description de la variation : Etudes transformationnelles des phrases du français de Côted'Ivoire. Thèse de Doctorat, Université de Grenoble 3.

Boutin, B. A. (2007). Déterminant zéro ou omission du déterminant en français de Côte d'Ivoire. Le français en Afrique, $\mathrm{n}^{\circ} 22,161-182$.

Boyer, H. (1996). Sociolinguistique : territoire et objets. Paris : Delachaux et Niestlé.

Bulot, T. (Dir.) (2004). Les parlers jeunes (Pratiques urbaines et sociales). Cahiers de Sociolinguistique 9, Rennes : PUR.

Bulot, T. et Ledegen G. (2008). Langues et espaces (Normes identitaires et urbanisation). Cahiers de Sociolinguistique (Nouvelle Série) 13, 5-14.

Calvet, L.-J. (1999). Pour une écologie des langues du monde. Paris : Plon.

Canut, C. (2000). Le nom des langues ou les métaphores des frontières. Ethnologies comparées, $\mathrm{n}^{\circ} 1$.

Chaudenson, R. (1981). Les langues véhiculaires. Paris : PUF.

Chaudenson, R. (1989). Créoles et enseignement du français. Paris : L’Harmattan.

Chaudenson, R. (1991). Le facteur urbain dans le devenir linguistique des pays africains. Le facteur linguistique dans la constitution des villes africaines. Cahiers des sciences humaines, vol 27, $\mathrm{n}^{\circ}$ 3-4, 305-313.

Chaudenson, R. (1992). Des îles, des hommes, des langues: essais sur la créolisation linguistique et culturelle. Paris: L'Harmattan.

Chaudenson, R. (1994). L'argot. Paris : PUF.

Chaudenson, R. (1994). Les voix de la ville. Paris : Payot.

Chaudenson, R. (2000). Grille d'analyse des situations linguistiques. Mondialisation: la langue française a-t-elle encore un avenir?, [s.1.], Institut de la Francophonie/Diffusion Didier Érudition, 173-233.

Chaudenson, R. (2004). La créolisation : théories, applications et implications. Paris : Karthala.

Chia, E. (1990). The New Speech Forms of Rapidly growing City: Pidgin French and Camfranglais in Yaoundé. Annales de la Faculté des Lettres et Sciences Humaines, VI, 1, 102-127.

Corbeil, J.-C., (1984). Le « français régional ». CILL 9, 3-4, 31-44.

Daff, M. (1995). Le français mésolectal oral et écrit au Sénégal : approche sociolinguistique, linguistique et didactique. Thèse de Doctorat, Dakar, Université Cheikh Anta Diop.

Daff, M. (1998). Petite vitrine syntaxique du français oral en Afrique. Queffelec, A. (ed.). Alternances codiques et français parlé en Afrique. Aix-en-Provence : Publications de l'Université de Provence, 107-129.

Dalbera, J.-Ph. (2002). Le corpus entre données, analyse et théorie. Corpus, Numéro 1, [http://corpus.revues.org/document10.html], (consulté le 15 décembre 2003).

Diouf, J.-L. (1991). Quelles langues parlent-ils à Dakar?. Des langues et des villes. Paris, Didier Érudition, 227-236.

Dreyfus, M. (1995). Le plurilinguisme à Dakar. Contribution à une sociolinguistique urbaine. Thèse nouveau régime. Paris : Université René Descartes-Paris V.

Dumond, P. (1991). Le français, une langue africaine. Paris : L'Harmattan.

Dumond, P. (1995). Sociolinguistique du français en Afrique francophone. Paris, EDICEF-AUPELF.

Echu, G. (2001). Le camfranglais : l'aventure de l'anglais en contexte multilingue camerounais. Ecritures, VII, 207221.

Edema, A.-B. (2006). L'hindoubill a-t-il été un laboratoire des particularismes lexicaux du français de Kinshasa ?. Le français en Afrique, $\mathrm{n}^{\circ} 21,17-40$.

Essono J.- M. (1997). Le camfranglais : un code excentrique, une appropriation vernaculaire du français. Le corpus lexicographique, Méthodes de constitution et de gestion, 381-396.

Féral, C. de. (1998). Français oral et camfranglais dans le sud du Cameroun. Queffélec A. (éd.). Alternances codiques et français parlé en Afrique. Aix-en-Provence : Publications de l'Université de Provence, 205-212.

Féral, C. de. (2004). Français et langues en contact chez les jeunes en milieu urbain : vers de nouvelles identités. In Penser la francophonie : concepts, actions et outils linguistiques. Paris : Editions des Archives Contemporaines, 583-597. 
Féral, C. de. (2006). Étudier le camfranglais : recueil des données et transcription. Le Français en Afrique, $n^{\circ} 21$, 211-218.

Feussi, V. (2006). Une construction du français à Douala-Cameroun. Thèse de doctorat unique. Tours : Université de Tours.

Feussi, V. (2008). Parles-tu français, ça dépend : penser-agir-construire son français en contexte plurilingue, le cas du Douala au Cameroun. Paris : L'Harmattan.

Fosso, (1999). Le camfranglais : une praxéogénie complexe et iconoclaste. In Mendo Ze, G. Le français langue africaine : enjeux et atouts pour la francophonie. Paris : Publisud, 178-194.

François-Geiger, D. (1989). L'Argoterie. Paris : Sorbonnargot.

Goffman, E. (1973). La mise en scène de la vie quotidienne. La présentation de soi. Paris : Minuit.

Goudaillier J.-P. (2003). Comment tu tchatches !, Paris, Maisonneuve \& Larose.

Gouédan, L. (1988). Les particularités lexicales dans un parler franco-ivoirien national : le nouchi. Mémoire de maîtrise, Université de Paris V.

Grafmeyer, Y. \& Joseph, I. (eds.) (1984). L'école de Chicago. Naissance de l'écologie urbaine. Paris : Aubier.

Gumperz, J. J. (1989). Sociolinguistique interactionnelle. Une approche interprétative. Paris : L'Harmattan.

Hall, E (1978). La dimension cachée. Paris : Points.

Harter, A.-F. (2007). Représentations autour d'un parler jeune : le camfranglais. Le français en Afrique, $\mathrm{n}^{\circ} 22,253-$ 266.

Hattiger, J.-L. (1981). Morpho-syntaxe du groupe nominal dans un corpus de français populaire d'Abidjan. Thèse de 3ème cycle. Strasbourg : Université de Strasbourg.

Hattiger, J.-L. (1983). Le français populaire d'Abidjan: un cas de pidginisation. Abidjan : ILA.

Jabet, M. (2006). Noms sans déterminants en français abidjanais: traits sociolinguistique, sémantiques et/ou pragmatique. Le français en Afrique, $\mathrm{n}^{\circ} 21,325-337$.

Kiessling, R. (2005). 'bak mwa me do' - Camfranglais in Cameroon. Lingua Posnaniensis, 47, pp. 87-107.

Knutsen, A.M. (2002). Le statut de là en français abidjanais. Romansk Forum, Nr. 16, 553-559.

Knutsen, A.M. (2007). Variation du français à Abidjan (Côte d'Ivoire). Etude d'un continuum linguistique et social. Thèse de doctorat. Oslo : Université d'Oslo.

Kouadio, N. J. (1992). Le nouchi abidjanais, naissance d'un argot ou mode linguistique passagère?. Des villes et des langues, actes du colloque de Dakar, Paris : Didier Érudition.

Kouadio, N. J. (2006). Le nouchi et les rapports dioula-français ». Le français en Afrique, $\mathrm{n}^{\circ}$ 21, pp. 177-192.

Kube, S. (2002). Das Nouchi in Abidjan: Vom Argot der Straßenkinder zur zukünftigen Nationalsprache der Côte d'Ivoire?. Osnabrücker Beiträge zur Sprachtheorie, n ${ }^{\circ}$ 65, 131-153.

Kube, S. (2005). La Francophonie vécue en Côte d'Ivoire. Paris : L'Harmattan.

Lafage, S. (1978). Rôle et place du français dans le continuum langues africaines/français de cote d'Ivoire. CIRL, $n^{\circ} 4, \mathrm{pp} 78-89$.

Lafage, S. (1980). Petite enquête sur la perception du français populaire ivoirien en milieu estudiantin. Bulletin CEP, $\mathrm{n}^{\circ} 6,1-37$.

Lafage, S. (1991). L'argot des jeunes ivoiriens, marque d'appropriation du français?. Langue française, $n^{\circ}$ 90, 95105.

Lafage, S. (1998). Hybridation et français des rues à Abidjan. In Queffélec A. (éd.), Alternances codiques et français parlé en Afrique, Aix-en-Provence : Publications de l'Université de Provence, 279-291.

Lafage, S. (2002). Le lexique français de Côte d'Ivoire, appropriation et créativité. Le français en Afrique, vol. 16.

Laroussi, F. et Babault, S. (2001). Variations et dynamisme du français : Une approche polynomique de l'espace francophone. Paris : L'Harmattan.

Lauter, B. et al. (1991). L'Etat et l'informel. Paris : L'Harmattan.

Léglise, I., Caubet, D., Billiez J. et al. (éds) (2004). Parlers jeunes, ici et là-bas. Pratiques et représentations. Paris : L'Harmattan.

Leimdorfer, F. et al. (2002). L'Afrique des citadins (Abidjan, Dakar). Sociétés civiles en chantiers. Paris : Karthala.

Lescutier, J-M. (1985). Recherches sur le processus de réactivation : cas singulier d'un idiolecte relevant du français populaire d'Abidjan. Université de Nice, Thèse de troisième cycle.

Maffesoli, M. (1979). La conquête du présent. Pour une sociologie de la vie quotidienne. Paris : PUF.

Manessy, G. (1994). L'appropriation du français en Afrique noire une dynamique discursive. Langue française, 104, $115-124$

Manessy, G. (1979). Le français en Afrique noire: faits et hypothèses. In Valdman, A. (ed), Le français hors de France, Paris : Champion, 333-362.

Manessy, G. (1989). Dire que... (De l'origine des créoles atlantiques). La linguistique, vol 25, fasc 2, 19-43.

Manessy, G. (1995). Créoles, pidgins, variétés véhiculaires. Procès et Genèse. Paris : Éditions du CNRS.

Manessy, G. et Wald, P. (1984). Le français en Afrique noire, tel qu'on le parle, tel qu'on le dit. Paris : L'Harmattan.

Marcellesi, J.-B. et Gardin B. (1974). Introduction à la socio-linguistique. La linguistique sociale. Paris : Larousse. 
Martinet, A. (1975). Evolution des langues et reconstruction. Paris : PUF.

Mendo-Ze, G. (1997). Le français langue camerounaise: enjeux pour la francophonie ; éléments de stratégie. Paris: Publisud.

Meunier-Crespo, M. (1994). Des données empiriques à l'objet d'étude: la constitution d'un corpus démodialectologique (Français populaire d'Abidjan). Lyon : CEL Université Jean Moulin.

Mufwene, S. S (2001). The Ecology of Language Evolution. Cambridge: Cambridge University Press.

Mufwene, S. S (2002). Competition and selection in language. Selection, 3, 45-56.

Mufwene, S. S (2005). Créoles, écologie sociale, évolution linguistique. Paris : L'Harmattan.

Mufwene, S. S (2007). Population movements and contacts in language evolution. Journal of Language Contact, 1, 63-91.

Myers-Scotton, C. (1993). Duelling languages: grammatical structure un codeswitching. Oxford: Clarendon Press.

Napon, A. (1992). Etude du français des non-lettrés au Burkina-Faso. Thèse de doctorat, Université de Rouen.

Ndao, P. A. (1996). Contact de langues au Sénégal. Étude du code switching wolof français en milieu urbain : approches sociolinguistiques, linguistiques et pragmatique. Thèse de doctorat d'État. Dakar: Université Cheikh Anta Diop.

Ngalasso, M. et al. (1998). «Le français des écoliers abidjanais : la revanche de la rue sur l'école ». Batiana, A. B. (eds). Francophonies africaines. Rouen, Dyalang, 49-65.

Nicolaï, R. (ed.). (2001). Leçons d'Afrique. Filiations, ruptures et reconstitution de langues. Un hommage à Gabriel Manessy. Louvain-Paris : Peeters.

Ntsobé, A. M., Biloa, E., Echu, G. (2008). Le camfranglais : quelle parlure ? Etude linguistique et sociolinguistique. Frankfurt : Peter Lang.

Ploog, K. (2002). Le français à Abidjan. Pour une approche syntaxique du non-standard. Paris: CNRS Editions.

Ploog, K. (2006). Du continuum pragmatico-sémantique aux types prosodiques de là en français abidjanais. Le français en Afrique, $\mathrm{n}^{\circ} 21,303-323$.

Ploog, K. (2007). Pour une approche comparative des dynamiques structurelles du français en Afrique. Actes du colloque franco-allemand, 1-8.

Poplack, S., (1988). Conséquences linguistiques du contact de langues: un modèle d'analyse variationniste. Langage et société, 43, 23-48.

Queffélec, A. (1998). Alternances codiques et français parlé en Afrique. Aix-en-Provence : Publications de l'Université de Provence.

Queffélec, A. (2000). Emprunt ou xénisme : les apories d'une dichotomie introuvable ?. In Latin, D. et Poirier, C. (dirs.). Contacts de langues et identités culturelles, Laval : Presses universitaires de Laval, 283-300.

Queffélec, A. (2007). Le camfranglais, un parler jeune en évolution : du résolecte au véhiculaire urbain. In Ledegen, G., Pratiques linguistiques des jeunes en terrains plurilingues, Paris : L'Harmattan, 93-118.

Queffélec, A. (2007). Les parlers mixtes en Afrique francophone subsaharienne. Le français en Afrique, $n^{\circ} 22,277$ 292.

Queffélec, A. (2008). L'évolution du français en Afrique noire, pistes de recherche. In Holter, K. et Skattum, I. (éds.). La francophonie aujourd'hui. Réflexions critiques, Paris : L'Harmattan, 63-76.

Queffélec, A. (2009). Alternances codiques et parlers hybrides en francophonie : convergences et divergences. Actes de colloque de Damas-Syrie, 171-180. [http://www.dlf.auf.org]. Consulté le 20/12.2009.

Robillard, D. (de) (1998). Langues, îles, simplicité, déterminisme, chaos. Plurilinguismes, n¹5, 48-66.

Robillard, D. (de) (2000). Villes, îles (socio) linguistiques. Des fenêtres sur une linguistique chaotique. In Calvet, L.J. et al.(éd.). Le plurilinguisme urbain. Paris : Didier, 463-480.

Robillard, D. (de) (2001). Peut-on construire des faits linguistiques comme chaotiques. Marges Linguistiques, $\mathrm{n}^{\circ} 1$, [http//www.marges.linguistiques.com]. Consulté le 10/10/2009.

Robillard, D. (de) (2007). La linguistique autrement: altérité, expérimentation, réflexivité, constructivisme, multiversalité : en attendant que le Titanic ne coule pas. $C A S, \mathrm{n}^{\circ} 1,1-148$.

Schroder, A. (2003). Status, Functions, and Prospects of Pidgin English. Tubingen: Gunter Narr Verlag.

Sesep N'sial, B. N. (1979). Le Métissage français-lingala au Zaïre. Essai d'analyse différentielle et sociolinguistique de la communication bilingue. Thèse de Doctorat. Nice : Université de Nice.

Swigart, L. (1992). Practice and Perception: Language Use and Attitudes in Dakar. Thesis. Washington: Washington University.

Thiam, N. (1994). La variation sociolinguistique du code mixte wolof français à Dakar : une première approche. Langage et Société, 68, 11-32.

Valdman, A. (1978). Le créole : structure, statut et origine. Genève : Klincksiek.

Wald, P. (1990). Catégories de locuteur et catégories de langue. Langage et société, n52, MSH, Paris, 5-22.

Wald, P. et Manessy, G. (1984). Le français en Afrique. Tel qu'on le parle, tel qu'on le dit. Paris : L'Harmattan.

Wenezoui-Déchamps, M. (1981). Le français, le sango et les autres langues centrafricaines. Enquête sociolinguistique au quartier Boy-Rabe (Bangui, Centrafrique). Paris : SELAF. 
Wenezoui-Déchamps, M. (1988). Entre langue coloniale et langue nationale: le franc-sango des étudiants de Bangui. Lengas, $n^{\circ} 23,25-35$.

Wenezoui-Déchamps, M. (1994). Que devient le français quand une langue nationale s'impose? Conditions et formes d'appropriation du français en République Centrafricaine. Langue française, ${ }^{\circ}$ 104, 89-99.

Wenezoui-Déchamps, M. (1995). Le franc-sango des «kota-zo» de Bangui: un exemple d'intégration des mots français dans un discours en langue africaine. ROFCAN, $\mathrm{n}^{\circ} 10,143-155$.

Wijnands, P. (2005). Le français adultère ou les français mixtes de l'altérité francophone. Paris : Publibook.

${ }^{1}$ Ces dénominations sont plus réelles pour les linguistes que pour les locuteurs.

${ }^{2}$ Tout ceci semble, à mon sens, approximatif du fait de la difficulté à tracer une frontière linguistique entre les FPA multiples et variès : où s'arrête un FPA, où commence un autre.

${ }^{3}$ Les concepts de créole/créolisation et de pidgin/pidginisation ont des définitions qui varient d'un point de vue à un autre, de la créolistique aux études francophones. Outre les débats classiques aux allures inslarocentrique, les créoles, langues nées des situations de contact depuis le $18^{\text {ème }}$ siècle, évoluent de la même manière que les autres parlers. Ils ont les mêmes processufs évolutifs qui peuvent être observables en dehors des espaces insulaires «lithurgiques ». Mais pas à décréter "créole» toute langue. C'est en cela que la créolistique peut apporter «l'évangile " à la linguistique contemporaine du fait de la spécificité légendaire de l'écosystème des créoles classiques. 